\title{
Sul gruppo concentrato di intersezioni di ipersuperficie algebriche.
}

\author{
Nota di Cesarina Marchionna Tibilettr (a Ferrara) \\ Ad Enrico Bompiani in occasione del suo Giubileo scientifico.
}

Sunto. - Vedi il primo capoverso del n. 1.

1. In questo lavoro si estende il concetto di «gruppo concentrato di interserzioni» dal caso relativo a dne curve algebriche piane, già ampiamente trattato in lavori precedenti (1), a quello di $r$ ipersuperficie immerse in un $S$, proiettivo complesso.

I risultati che ora indicheremo sono già stati annunciati in una recente conferenza $\left({ }^{2}\right)$ senza però riferire sulle relative dimostrazioni. Scopo principale di questa nota è appunto quello di esporre per disteso tali dimostrazioni che, rispetto al caso piano, talvolta sono delle immediate estensioni e talaltra sono di tipo diverso o più complicate.

2. Consideriamo $r$ ipersuperficie algebriche $F_{j}=0(j=1, \ldots, r)$ immerse in uno spazio proiettivo complesso $S_{r}$ (con $r \geq 2$ ), ove sia fissato un sistema di coordinate proiettive omogenee $x_{h}(h=0, . ., r)$. Supporremo sempre che le intersezioni di tali $F_{j}=0$ siano date solo da punti isolati.

Variamo poi ciascuna $F_{j}=0$ sostituendo rispettivamente ogni coeffi. ciante della sua equazione con una funzione analitica di un parametro (complesso) $\varepsilon$, la quale, in $\varepsilon=0$, abbia i valori del corrispondente coefficiente della $F_{j}=0$ considerata. Una variazione di questo tipo verrà detta regolare so: 1) ipersuperficie variate banno solo intersezioni (isolate e) semplici nell' intorno di $\varepsilon=0$; 2) i coefficienti delle equazioni delle ipersuperficie variate sono delle funzioni anatitiche (della variabile $\varepsilon$ ) sprovviste di singolarità in $\varepsilon=0$.

Nella presente nota considereremo solo (anche quando non lo ripeteremo esplicitamente) variazioni regolari delle $F_{j}=0 \quad(j=0, \ldots, r)$ del tipo ora definito.

In particolare le ipersuperficie $F_{j}=0$ posseggano riunite in un certo punto $P$ (semplice o multiplo in un modo qualsiasi sulle $F_{j}=0$ ) $i$ (e solo j) intersezioni.

(1) Cfr. [6], [7], [8].

(2) Cfr. [9]. 
Sia ora $F_{j}^{\varepsilon}=0$ l'ipersuperficie ottenuta variando la $F_{j}=0$ (nel modo suddetto) $e$ si indichino con $P_{k}(k=1, \ldots, i)$ le $i$ intersezioni semplici delle $F_{j}^{\varepsilon}=0$ che quando $\varepsilon \rightarrow 0$ confluiscono in $P$.

Infine consideriamo un sistema di $i$ equazioni lineari indipendenti che traducono le condizioni di passaggio di una ipersuperficie algebrica (di un certo ordine abbastanza elevato) per $i$ punti $P_{k}$; indicheremo con il simbolo $S_{\varepsilon}$ l'insieme di tutte le equazioni che risultano combinazioni lineari delle equazioni contenute nel sistema suddetto (naturalmente tale insieme può essere generato da sistemi diversi di $i$ equazioni indipendenti).

3. Precisiamo anzitutto la natura delle suddette condizioni $S_{\varepsilon}$ e poi per esse dimostriamo alcune proprietà, analoghe a quelle già dimostrate in [6] per il caso piano.

Supponiamo (e ciò non altera la natura del problema in esame) che il punto $P$ sia l'origine $O$ del sistema di riferimento.

Le coordinate $x_{h}^{(k)}(h=0, \ldots, r)$ dei suddetti punti $P_{k}(k=1, \ldots, i)$, distinti, saranno date da serie convergenti nell' intorno di $\varepsilon=0$ del tipo

$$
x_{h}^{(k\rangle}=\sum_{l=1}^{\infty} \alpha_{h l}^{\langle k\rangle} \varepsilon^{l} \quad(h=0, \ldots, r ; k=1, \ldots, i),
$$

ove i coefficienti $\alpha_{h l}^{(k)}$ non dipendono da $\varepsilon$.

Veramente, a priori, le coordinate $x_{h}^{(h)}$ dei punti $P_{k}$ potrebbero essere date da serie di Puiseaux in $\varepsilon$ e cioè da serie di Taylor in $\varepsilon^{\frac{1}{m}}$ con $m>1$, intero: però, se ciò accadesse, basterebbe porre $\varepsilon$ in luogo di $\varepsilon^{\frac{1}{m}}$ (tutte le volte che si presentasse una tale eventualità).

Le (1) sono tali che posti gli $x_{h}^{\langle k\rangle}$ in luogo di $x_{h}$ nelle equazioni delle $F_{j}^{\varepsilon}=0(j=1, \ldots, r)$ queste risultano identicamente soddisfatte.

Imponiamo ora ad una ipersuperficie di ordine $n$ abbastanza elevato (preciseremo poi quanto basta) di equazione

$$
\Sigma \alpha_{i_{0} \ldots i_{r}} x_{0}^{i_{u} \ldots} x_{r}^{i r}=0 \quad\left(\text { con } i_{0}+\ldots+i_{r}=n\right)
$$

di passare per i punti $P_{k}$.

Questo fatto importa appunto per i coefficienti $a_{i_{0}} \cdots i_{r}$ l'insieme $S_{\varepsilon}$ di equazioni lineari indicate sopra. Pertanto nelle equazioni che costituiscono $S_{\varepsilon}$ le incognite sono le $a_{i_{0}} \ldots_{i_{r}}$ ed $\mathrm{i}$ coefficienti (delle $a_{v_{0}} \ldots i_{r}$ ) possono essere dati da funzioni analitiche $\varepsilon$ regolari nell'intorno di $\varepsilon=0$.

Come è ben noto, quando $n$ è elevato $\left(n>i\right.$, per esempio) in $S_{\varepsilon}$ sono 
contenuti corti grappi di $i$ (e non più di $i$ ) equazioni fra loro indipendenti ( $\theta$ ciò per $\varepsilon \neq 0$ appartenente ad un intorno di $\varepsilon=0$ ).

Notiamo ancora che un qualsiasi sistema lineare omogeneo di $i$ equazioni indipendenti estratto da $S_{\dot{z}}$, ha soluzioni che (tramite i minori estratti dalla matrice dei coefficienti del sistema stesso) possono essere senz'altro rappresentate da funzioni analitiche di $\varepsilon$, regolari nell'intorno di $\varepsilon=0$.

Pertanto, un' ipersuperficie $F_{\varepsilon}=0$ (di ordine $n$ ) che ha come coefficienti una di tali soluzioni di $S_{\varepsilon}$ per $\varepsilon \rightarrow 0$ tende ad una ipersuperficie $F=0$, passante per $P$ i cui coefficienti soddisfano alle condizioni limiti (per $\varepsilon \rightarrow 0$ ) delle $S_{\varepsilon}$ stesse, che indichiamo con $S_{P}^{i}$.

Ciò posto, dimostriamo i seguenti teoremi.

Teorema 1. L'insieme delle equazioni $S_{s}$ (relative al punto $P T$ ) al limite, per $\varepsilon \rightarrow 0$, diventa un insieme $S_{P}^{i}$ di equazioni lineari che contiene ancora $\mathrm{i}$ equazioni indipendenti $\left({ }^{3}\right)$.

Le equazioni lineari (nelle $\mathrm{N}+1$ variabili omogenee $a_{i_{0} \ldots i_{r}}$ ) che costituiscono l'insieme $S_{\varepsilon}$ possono essere interpretate come equazioni di iperpiani in uno spazio lineare $\Sigma_{N}$ ad $N$ dimensioni ove orviamente $N>i$; chiamiamo $\pi_{\varepsilon}$ tale insieme di iperpiani. Poichè $S_{\varepsilon}$ contiene sistemi di $i$ (e non più di $i$ ) equazioni indipendenti lo spazio comune a tutti gli iperpiani di $\pi_{\varepsilon}$ è un $\Delta_{\varepsilon}$ di dimensione $N-i$.

Passiamo al limite per $\varepsilon \rightarrow 0$ : il sistema $\pi_{\varepsilon}$ tende ad un sistema $\pi$ di iperpiani (anzi ogni iperpiano di $\pi_{\varepsilon}$ ha per limite un iperpiano di $\pi\left({ }^{+}\right)$). A priori il sistema $\pi$ ha dimensione $\mu \leq i-1$, essendo $i-1$ la dimensione di $\pi_{\varepsilon}$ ). Lo spazio $\Delta$ (limite di $\Delta_{\varepsilon}$ ) che $\partial$ comune a tutti gli iperpiani di $\pi$ avrà dimensione $N-i+h$ con $h \geq 0$.

Supponiamo dapprima che sia $h>0$. In tale ipotesi fissiamo $i$ gruppi $G_{s}(s=1, \ldots, i)$ formati ciascuno da $i-1$ punti con coordinate che non (lipendono da $\varepsilon$ e generici nel senso che: 1) i gruppi $G_{s}$ e $\Delta_{\varepsilon}$ definiscono rispettivamente (per $s=1, \ldots, i) \quad i$ iperpiani (di $\pi_{\varepsilon}$ ) fra loro indipendenti; 2) ogni $G_{s}$ e $\Delta$ non sono contenuti in uno stesso iperpiano (5).

(3) La dimostrarione di questo teorema è simile a quella data in [6], $\$ 3$ per l'analoga proprietà piana, però la riferiamo per disteso, per ragioni di chiarenza. data la sua impor* tanza nello svolgimento della presente nota.

( $\left.{ }^{\star}\right)$ Notiamo che ogni iperpiano $\alpha_{\varepsilon}$ di $\pi_{\varepsilon}$ ha per limite un iperpiano $\alpha$ di $\pi$ in quanto i coefficienti dell' equazione di $\alpha_{\varepsilon}$ sono (nell'intorno di $\varepsilon=0$ ) funzioni analitiche regolari (serie di potenze) in $\varepsilon$. Naturalmente se $i$ coefficienti di tale equazione sono tutti divisibili per $\varepsilon^{s}$ e non per $\varepsilon^{i+1}$ si arrà eura di passare al límite dopo aver diviso per $\varepsilon^{s}$ entrambi $i$ membri dell' equazione di $\alpha_{\varepsilon}$.

${ }^{5}$ Ciò è possibile poichè qui supponiamo che $\Delta$ abbia dimensione $N-i+h$ con $h>0$. 
Per chiarezza possiamo supporre $N$ sufficientemente alto in modo che $\mathrm{i}$ punti dei grappi $G_{s}$ siano dati da punti fondamentali di $\Sigma_{N}$, anche tutti diversi fra loro.

Consideriamo poi gli $i$ iperpiani $\pi_{s}^{\varepsilon}(=1, \ldots, i)$ indipendenti di $\pi_{s}$ passanti rispettivamente per i $G_{s}$. Passiamo ora al limite per $\varepsilon \rightarrow 0$; gli iperpiani $\pi_{s}^{\varepsilon}$ tenderanno rispettivamente a dei piani $\pi_{s^{\prime}}(s=1, \ldots, i)$ che passano ancora, rispettivamente, per i gruppi $G_{s}$ (la cosa è particolarmente evidente se i punti dei $G_{s}$ sono dati da punti fondamentali di $\Sigma_{N}$, come è detto sopra).

Tali iperpiani $\pi_{s}$ passano nataralmente per $\Delta$ e quindi contro le ipotesi attuali avremmo degli iperpiani per $\Delta$ e per $\mathbf{i} G_{s}$.

Si giunge cosi ad un assurdo per $h>0$, e quindi $\Delta$ ha sempre dimensione $N-i$, il sistema limite $\pi$ ha dimensione $i-1$ ed $S_{P}^{i}$ contiene $i$ equazioni indipendenti. È così completamente dimostrato il teorema 1.

Teonema 2. Le ipersuperficie che in $P$ hanno un punto multiplo di ordine $\geq i$ verificano senz'altro le. $S_{P}^{i}$ limiti di un insieme $S_{\varepsilon}$, ottenuto in relazione ad una variazione regolare (del tipo sopra indicato).

Consideriamo $i+h$ (con $h \geq 0$ ) iperpiani qualsiansi, distinti o coincidenti di $S_{r}$, eiascuno parallelo ad un iperpiano di riferimento, passanti tutti per i punti $P_{k}$, in modo che per ogni punto $P_{k}$ ne passi qualenno.

Supponiamo sempre che $P$ sia l'origine 0 : allora per $\varepsilon \rightarrow 0$ l'insieme dei suddetti iperpiani diventa un'ipersuperficie di equazione

$$
x_{1}^{h_{1}} x_{2}^{h_{2}} \ldots x_{r}^{h_{r}}=0
$$

(ove $x_{1}, \ldots, x_{r}$ sono ora. le coordinate non omogenee in $S_{r}$ ), con $h_{1}+h_{2}+\ldots+h_{v}=i+h \quad\left(\right.$ ed $\left.h_{\imath} \geq 0\right)$.

Pertanto, per le prime proprietà-riferite all'inizio del presente paragrafo, le ipersuperficie aventi equazioni del tipo (3) verificano le $\mathrm{S}_{P}^{i}$ (in quanto ipersuperficie limiti di ipersuperficie soddisfacenti alle $S_{\varepsilon}$ ).

Verificano le condizioni lineari $S_{P}^{i}$ anche le ipersuperficie di equazione

$$
\Phi_{i+n}\left(x_{1}, \ldots, x_{r}\right)=0
$$

ove $\Phi_{i+h}\left(x_{1}, \ldots, x_{r}\right)$ ¿े un polinomio omogeneo nelle $x_{1}, \ldots, x_{r}$ di grado $i+h(\operatorname{con} h \geq 0)$.

Quindi soddisfano alle $S_{P}^{i}$ tutte le ipersuperficie di equazione

$$
\Phi_{i}\left(x_{1}, \ldots, x_{r}\right)+\Phi_{i+1}\left(x_{1}, \ldots, x_{r}\right)+. .+\Phi_{i+q}\left(x_{1}, \ldots, x_{r}\right)=0
$$

ciò̀ tutte le ipersuperficie che hanno in $P$ un punto multiplo di ordine $\geq i$. 
Teorema 3. Le $S_{P}^{i}$ sono indipendenti dal tipo di variazione (regolare) impiegato yer passare dalle $F_{j}=0$ a delle $F_{j}^{\varepsilon}=0$.

La dimostrazione si conduce come quella data in [6] per il teorema 6 . Qui però si usa il teorema di Noether-Severr ( $\left.{ }^{6}\right)$ in luogo del teorema di NoEther relativo alle curve piane.

Inoltre serve una proposizione analoga a quella data in [6] col Teorema 2, ma in relazione ad un polinomio di più variabili invece che ad uno di due variabili ('). Anche quest' ultima proprietà si dimostra estendendo banalmente quanto è detto in [6] per i Teoremi 1 e 2.

Le proprietà ora dimostrate si riassumono nella seguente proposizione: «Siano date $r$ ipersuperficie algebriche $F_{j}=0(j=1, \ldots, r)$ immerse in uno spazio $S_{r}$, aventi in comune solo dei punti isolati. Ad ogni punto $P$, in cui siano riunite $i$ intersezioni delle $F_{j}=0$ è legato un insieme di condizioni lineari $S_{P}^{i} d a$ cui se ne possono estrarre gruppi $d i \quad i$ e non piu di $i$ indipendenti. Queste sono lamiti di condizioni di passaggio di un'ipersuperficie (di ordine $n$ abbastanza elevato) per certi punti $P_{k}$ (comuni ad $r$ ipersu. perficie $F^{\varepsilon_{j}}=0(j=1, \ldots, r)$ che, quando $\varepsilon \rightarrow 0$, con una qualsiasi variazione regolare, diventano rispettivamente le $F_{j}=0$ : i punti $P_{k}$ sono esattamente le intersezioni - semplici ed isolate - delle $F_{i}=0$ che cadono in $P$ per $\varepsilon \rightarrow 0) »$.

(6) Cfr. ad es. [1], pag. 311.

(7) Precisamente le proposizioni cui si allude sono le seguenti, immediate estensioni (per l'enunciato e la dimostrizione) dei teoremi 1 e 2 di [6]

I) $\mathrm{Si}$ indichi con $F$ un polinomio di $r$ variabili $x_{1}, \ldots, x_{r}$ e con $F s_{1}, \ldots, s_{r}$ la sua derivata di ordine $s_{1}+\ldots+s_{r}$ (ove $s_{i} \geq 0$ ) data da $\frac{\partial s_{1}+\ldots+s_{r} W}{\partial x_{s_{1}} \ldots \partial x_{s_{r}}}$ considerando la $F$ stessa come derivata di ordine zero $F_{0}, \ldots, 0=F$ (ovviamente, in generale un $s_{i}=0$ indica che non si deriva rispetto alla variabile $x_{i}$ ). Si fissino in uno spazio $S_{r}$ (ove $x_{1}, \ldots, x_{r}$ sono le coordinate non omogenee) $k$ punti $P j(j=1, \ldots, k)$ di coordinate $x_{1}(j), \ldots, x_{2}(j), \ldots, x_{2}(l)$, si consideri in particolare uno di essi $P_{1}$, si diano in relazione a ciascuno dei punti $P_{j}$ certi numeri interi positivi $n_{j}(j=1,2, \ldots, k)$ e in relazione a $P_{1}$ anche $r$ numeri interi $\sigma_{1}, \ldots, \sigma_{r}$ con $\sigma_{l} \geq 0$ $(l=1, \ldots, r)$ e $\underset{1}{\Sigma_{l}} \sigma_{l}<n_{1}$. Si può allora costruire un polinomio di grado abbastanza elevato che soddisfi alle seguenti, condizioni: 1) in $P_{1}$ siano nulle tutte le derirate $F s_{1}, \ldots, s_{r}$ con $\underset{1}{\Sigma_{h}} s_{h}<n_{1}$ ad eccezione della $\left.F_{\sigma_{1}}, \ldots, \sigma_{r} ; 2\right)$ in ciascun altro punto $P_{j}$ siano nnlle tutte le $F_{s_{1}}, \ldots, s_{r}$ per cui si ha rispettivamente, $\underset{1}{r} s_{h} s_{h}<n_{j}$.

II) Assegnato un certo gruppo di punti in uno spazio $S_{r}$ (ove è fissato un sistema di coordinate non omogenee $\left.x_{1}, \ldots, x_{r}\right)$ è possibile costituire un polinomio $F\left(x_{1}, \ldots, x_{r}\right)$ nelle $r$ variabili $x_{1}, \ldots, x_{r}$, di ordine abbastanza alto, tale che in ciasenno dei punti dati esso e le sue derivate (fino ad un ordine prefissato - per ciascun punto -) assumano valori assegnati. 
4. Usiamo ora in $S_{r}$, le coordinate non omogenee $x_{1}, x_{2}, \ldots, x_{r}$. Diremo che in un punto $P$ isolato comane ad $r$ ipersuperficie $F_{j}=0(j=1, \ldots, r)$ di $S_{r}$, unx ipersuperficie $F=0$ verifica la condizione $d i$ NoETHER quando è possibile scrivere nell' intorno di $P$

$$
F=\sum_{1}^{r} A_{h}^{\prime} F_{h}
$$

ove le $A_{h}^{\prime}$ sono serie di potenze, anche solo formali $\left({ }^{s}\right)$, in $r$ variabili, nel punto $P$ stesso.

Si dimostra in modo del tutto analogo a quello usato in [6] per il teorema 9 il seguente teorema 4 (preliminarmente si verificano proprieta simili a quelle date dai lemmi 7 ed 8 della [6], e ció come nella stessa [6].

Teorema 4. Se una ipersuperficie $F=0$ soddisfa alla condizione di Noether nel punto $P$, essa verifica le $S_{P}^{i}$ (relative a $P$ ) e viceversa.

5. Infine possiamo concludere, come nel caso piano con le seguenti proposizioni.

Siano date le suddette ipersuperficie $\mathbf{F},=0$, immerse in $S_{r}$ che pos. seggano $i$ intersezioni riunite in un punto $P$.

Diciamo che «queste ipersuperficie $F_{j}=0(j=1, \ldots, v)$ posseggono un gruppo concentrato di $i$ intersezioni in $P$ e che un ipersuperficie $F=0$ passa per lo stesso gruppo concentrato di intersezioni quando soddisfa alle precedenti condizioni lineari $S_{P}^{i}$ (relative allo stesso punto $P$ )》.

Questo concetto di «gruppo concentrato di punti comuni alle $F_{j}=0$ » è definito qui in forma astratta e la sua introduzione è giustificata, come nel caso piano $\left({ }^{9}\right)$, dal fatto che esso soddisfa alle proprietà fondamentali delle intersezioni semplici di $r$ ipersuperficie (e ciò in accordo col principio di permanenza delle proprietà formali).

Precisamente un gruppo $\mathbf{I}$ di $i$ intersezioni, di $r$ ipersuperficie $F_{s}=0$, concentrate in un punto $P$ soddisfa alle seguenti quattro proprietà.

(8) Si dimostra che quando vi è questa possibilità per $F$ si può anche scrivere $F$ in una forma del tipo (4) in cui però le $A_{h}^{\prime}$ sono serie di potenze convergenti nell'intorno di $P$.

La dimostrazione di questo fatto si ottiene estendendo in modo ovvio quella data in [6], \$ 6. Por tale estensione occorre però ricordare la seguente proposizione. "Date le precedenti $E_{j}=0$ (nelle variabili non omogenee $x_{1}, \ldots, x_{h}$ ), è sempre possibile trovare un polinomio $R\left(x_{k}\right)$ nella sola variabile $x_{h}$ per eui si abbia

$$
R\left(x_{h}\right)={\underset{1}{\Sigma}{ }_{i}^{\prime} A_{l} F_{l}}_{l}^{r} \quad(h=1, \ldots, r)
$$

con $A_{l}$ polinomio nelle $x_{1}, \ldots, x_{r} \gg$. Quest' ultimo fatto, ben noto, deriva immediatamente per esempio - dal teorema del n. 14l, a pag. 33̄̄, di F. SEveri, Lezioni di Analisi, Bologna 1943, vol. 1 .

${ }^{9}$ ) Cfr. [6], \$ 7 . 
a) Le condizioni di passaggio per $\Gamma$ sono date da equazioni lineari che risultano limiti di effettive condizioni di passaggio per punti distinti.

b) Tutte le ipersuperficie d'equazione $F=\sum_{h}^{r} A_{h} F_{h}$, ove gli $A_{h}$ sono dei polinomi qualsiansi (per esempio, nelle variabili non omogenee $x_{1}, \ldots, x_{r}$ ) passano per $\boldsymbol{\Gamma}$.

c) L'equazione $F=0$ di un'ipersuperficie che rassa per $\Gamma$ può sempre scriversi nell'intorno di $P$ nella forma $F=\sum_{1}^{r} A_{h}^{\prime} F_{h}$ ove le $A_{h}^{\prime}$ sono serie di potenze (di $r$ variabili) in $P$.

a) Le $r$ ipersuperficie $F_{j}=0$ abbiano in comune in an punto $P$ un gruppo concentrato $\Gamma$ di $i$ (e solo $i$ ) intersezioni; $r$ altre ipersuperficie $G_{j}=0(j=1, \ldots, r)$ passino per tale $\Gamma$ ed abbiano solo $i$ intersezioni riunite in $P$. Allora ogni altra ipersuperficie $H=0$, che nell' intorno di $P$ si può scrivere $H=\sum_{1}^{r} A_{h}^{\prime} F_{h}=0$ (con $A_{h}^{\prime}$ serie di potenze - in $r$ variabili - in $P)$ si può scrivere anche, nell'intorno di $P$, nella forma $H=\Sigma_{h}^{r} B_{h}^{\prime} G_{h}=0$ (con $B_{h}^{\prime}$ serie di potenze-in $r$ variabili - nello stesso punto ${ }^{\prime} P$ ). Cioè il gruppo $\Gamma$ \& caratterizzato da una qualsiasi $r$-pla di ipersuperficie che passano (in un modo qualunque) per $I$ ed hanno raccolte in $P$ solo $i$ intersezioni.

Le proposizioni $a), b$ ), c) derivano ovviamente dai teoremi riferiti sopra.

L'ultima proprietà d) si dimostra con considerazioni diverse da quelle usate a proposito dell' analoga proposizione piana $\left({ }^{10}\right)$.

Riferiamo una tale dimostrazione nel seguente n. 6 .

6. Diamo dunque la dimostrazione della proposizione $d$ ) del precedente n. 5 . Mostriamo dapprima che è possibile scrivere $F_{j}=0$ nella forma

$$
F_{j}=\sum_{1}^{r} L_{j h}^{\prime} G_{h}
$$

nell intorno di $P$, con $L_{j h}^{\prime}$ serie di potenze - del solito tipo - in $P$.

Per ipotesi è possibile scrivere

$$
G_{j}={\underset{1}{\Sigma}}_{n}^{r} C_{j h}^{\prime} F_{h}
$$

ove le $C_{3 n}^{\prime}$ sono serie di potenze in $r$ variabili convergenti in $P\left({ }^{11}\right)$.

(10) Cfr. [6], § 8.

(11) Cfr. la nota (8), relativa al n. 4. 
Sia ora $c_{j h}$ il termine noto di $C_{j h}^{\prime}$ : si ha

$$
G_{j}=\sum_{1}^{r}{ }_{1} c_{j h} F_{h}+\sum_{1}^{r} D_{j h}^{\prime} F_{h}
$$

ove le $D_{j h}^{\prime}$ sono serie di potenze convergenti in $P$ che si annullano nello stesso punto $P$.

Consideriamo il determinante

$$
\Omega=\left\|c_{j h}\right\|
$$

e mostriamo che, nelle nostre ipotesi, è $\boldsymbol{Q} \neq 0$.

Diamo una dimostrazione per assurdo supponendo $\boldsymbol{Q}=0$. In tale ipotesi una riga di $\Omega$ è combinazione lineare delle altre: per esempio

$$
c_{1 h}=\sum_{2}^{r} \lambda_{m} c_{m h} \quad(h=1, \ldots, r)
$$

Poniamo

$$
M_{j}=\sum_{1}^{r} c_{j h} F_{h} \text { ed } N_{s}=\sum_{1}^{r} D_{j h}^{\prime} F_{h}
$$

si ha

$$
G_{j}=M_{j}+N_{j}
$$

Ciò posto, dalle (6) si deduce

$$
\begin{gathered}
M_{1}=\sum_{1}^{\Sigma_{h}} c_{1 h} F_{h}=\underset{1}{\sum_{h}} \sum_{2}^{r} \Sigma_{m} \lambda_{m} c_{m h} F_{h}=\underset{2}{\sum_{m} \lambda_{m}} \underset{1}{\stackrel{r}{\Sigma}} c_{m h} F_{h}= \\
=\underset{2}{\Sigma_{m}} \lambda_{m} M_{m} .
\end{gathered}
$$

Allora

$$
G_{1}=M_{1}+N_{1}=\sum_{2}^{r} \lambda_{m} M_{m}+N_{1}=\sum_{2}^{r} \lambda_{m} G_{m}-\underset{i}{\stackrel{r}{r}} \lambda_{m} N_{m}+N_{1}
$$

Ora dalle (7) si ha

$$
N_{1}-\sum_{2}^{r} \lambda_{m} N_{m}={\underset{1}{\Sigma}}_{h}^{r} E_{h}^{\prime} F_{h}
$$

ove le $E_{h}^{\prime}$ sono serie di potenze (del solito tipo) che si annullano in $P$. 
C. Marchionna TibiLfrreI: Sul gruppo concentrato ai interseaioni, coc. 157

Infine, usando le (8) e (9), l'intersezione delle $G_{0}=0$ può essere data dal sistema

$$
\left\{\begin{array}{l}
\stackrel{r}{\Sigma}_{h} E_{n}^{\prime} F_{h}=0 \\
G_{2}=0 \\
\ldots \ldots \\
G_{r}=0
\end{array}\right.
$$

Di qui si deduce, tenendo conto delle (5) e ricordando che tutte le $E_{h}^{\prime}$ si annullano in $P$, che il numero delle intersezioni delle $G_{j}=0$ rac. colte in $P$ sono più $d i$.

Quindi sarà $\Omega \neq 0$ e dal sistema (5) è allora possibile ricavare delle relazioni.

$$
F_{j}=\underset{1}{\stackrel{r}{\Sigma}} L_{j h}^{\prime} G_{h}
$$

in cui le $L_{j h}^{\prime}$ sono serie di potenze nell' intorno di $P$.

Pertanto tenendo conto dell'ipotesi

$$
H=\sum_{1}^{r} A_{n}^{\prime} F_{h}
$$

e della (11) si ha

$$
H=\sum_{1}^{r} B_{h}^{\prime} G_{h}
$$

(con $B_{h}^{\prime}$ serie di potenze - del solito tipo - in $P$ ) e ciò come volevasi dimostrare.

7. Ricordiamo ancora che B. SEgRe nell' interessante memoria [2] $\left({ }^{12}\right)$ əे giunto a risultati che si collegano a quelli esposti nella presente nota.

Precisamente B. Segre determina con metodi diversi dai nostri (che utilizzano brillantemente la rappresentazione delle forme algebriche su una varietà di Veronese) delle condizioni locali topologico-differenziali le quali forniscono le condizioni di appartenenza di una forma ad un modulo. Queste ultime condizioni sono, press'a poco, come ha osservato lo stesso prof. SEGRE, un'estensione delle $S_{P}^{i}$ che avevamo già introdotto in [6] a proposito delle curve piane (e che ora abbiamo determinato in relazione ad un $S_{\text {, qualsiasi). }}$

(12) Cfr. anche [3]. 
Infine osserviamo che i risultati delle note [7] e [8] relativi al teorema dell' $A f+B \varphi$ e all' appartenenza di polinomi a moduli di polinomi in due variabili si possono estendere al caso di polinomi di più variabili.

Alcuni enunciati di tali estensioni sono riportati in [9], cap, V; altri si possono facilmente immaginare, insieme alle dimostrazioni, che si rifanno sulla falsariga di quelle esposte in [7] ed [8]; pertanto pensiamo opportuno non dilungarci oltre su tali questioni.

Ricordiamo ancora che, sfruttando i risultati sopra citati B. SEGRE ha pure dato (in [2]) eleganti soluzioni a proposito di problemi riguardanti appartenenze di forme a certi moduli.

\section{BIBLIOGRAFIA}

[1] E. Bertini, Geometria proiettiva degli iperspazi, Principato, llessina (1923).

[2] B. SEgre, Invarianti topologico differenziali, varietà di Veronese e moduli di forme algebriche, "Annali di Matem.» (IV) 41 (1956).

[3] - -, Some properties of differentiable varieties and transformations, etc., «Ergebnisse der Math., u. ihrer Grenzgebiete, Neue folge, heft 13, Springer, Berlin (1957).

[4] F. SEYERI, Rappresentazione di una forma qualunque per combinazione lineare di più altre, «Rend. Acc. Lincei » (5) 11 (1902) e Mem. scelte, I, Zuffi, Bologna (1950).

[5] - -, Su alcune questioni di postulazione, "Rend, Circ. Matem. di Palermos 17 (1903).

[6] C. TIвILetTi, Gruppo concentrato di intersezioni di due curve algebriche, "Rend. Ist. Lombardo 84 (1951).

[7] - - Il teorema generale dell' $A \varphi+B \psi$ dedotto da un compnto di costanti, "Rend. Ist. Lombardo», 84 (1951).

[8] - - Alcune estensioni di un teorema di Noether, "Rend. di Matem." e sne applicazioni 10 (1951).

[9] C. Marchionna Tibilettr, Groupe concentré d'intersections de courbes et hypersurfaces algébriques. Applications, $3^{\circ}$ Coll. de Géom. alg., Bruxelles (1959). 